\title{
Reactions of the Escherichia coli flavohaemoglobin (Hmp) with NADH and near-micromolar oxygen: oxygen affinity of NADH oxidase activity
}

\author{
Robert K. Poole, ${ }^{1}$ Nikolaos loannidis' + and Yutaka Orii ${ }^{2}$ \\ Author for correspondence: Robert K. Poole. Tel: +44 171333 4275. Fax: +44 1713334500. \\ e-mail: udbs065@bay.cc.kcl.ac.uk
}

1 Division of Life Sciences, King's College London, Campden Hill Road, London W8 7AH, UK

2 Department of Public Health, Graduate School of Medicine, Kyoto University, Kyoto 606, Japan

\begin{abstract}
The soluble flavohaemoglobin (Hmp) of Escherichia coli, product of the hmp gene, contains haem B and FAD in a single polypeptide of molecular mass $44 \mathrm{kDa}$. The function of this protein (and of the similar proteins identified in several bacteria and yeast) is unknown, but the observation that the binding of oxygen to haem modulates the reduction level of FAD has suggested that Hmp could act as an oxygen sensor. Here, stopped-flow, rapid-scan spectroscopy has shown that the oxidized protein reacts rapidly with NADH to form an oxygenated species, even when efforts are made to reduce oxygen concentrations to sub-micromolar levels, suggesting a high affinity for this ligand. As is the case at high oxygen concentrations (130 $\mu \mathrm{M})$, oxygenated species formation was kinetically and spectrally heterogeneous. Between $12 \mathrm{~ms}$ and $1 \mathrm{~s}$ after mixing, following transient formation of the deoxy form and its reaction with dioxygen, a steady-state level of the oxygenated species was attained. During the oxygenated steady state, the flavin remained largely oxidized, as observed previously at $130 \mu \mathrm{M}$ oxygen. Hmp is an NADH oxidase; on exhaustion of oxygen by reduction (in $<10 \mathrm{~s}$ under these conditions), the oxygenated species disappeared to generate the deoxy Fe(II) haem, whereupon the flavin was reduced. The affinity for oxygen during NADH oxidation was measured by continuous dual-wavelength monitoring of the deoxygenation of oxymyoglobin. The $K_{m}$ for oxygen was $2.6 \mu \mathrm{M}$, much higher than the $K_{m}$ values determined, using the same method, for the membranebound terminal oxidases cytochromes bo' and bd. These results show that the oxidase activity of Hmp, but not necessarily oxygen binding, would be minimal at oxygen concentrations that limit terminal oxidase function.
\end{abstract}

Keywords: haemoglobin (bacterial), Escherichia coli, Hmp, oxygen affinity, oxidase

\section{INTRODUCTION}

The existence of globin-like proteins in micro-organisms is now well-established. The first example of a bacterial haemoprotein shown to have the characteristics of a globin, in terms of amino acid sequence (Wakabayashi et al., 1986) and oxygen-binding characteristics (Orii \& Webster, 1986), was a soluble protein from Vitreoscilla (reviewed by Webster, 1987), originally referred to as a 'cytochrome o' (Webster \& Liu, 1974). This protein is

†Present address: NCSR 'Demokritos', Institute of Materials Science, 153 10 Ag. Paraskevi Attiki, Athens, Greece.
$26 \%$ identical to lupin leghaemoglobin and is clearly related to globins in higher organisms (Perutz, 1986). The function of Vitreoscilla haemoglobin $(\mathrm{Vgb})$ is unclear, but the elevated levels observed in hypoxic conditions (Boerman \& Webster, 1982), and the finding that multicopy expression of the cloned $v g b$ gene in Eschericbia coli enhances growth under oxygen-limited conditions (Khosla \& Bailey, 1988; Khosravi et al., 1990), suggest that it has an important function in delivering, or perhaps storing, oxygen.

Vasudevan et al. (1991) identified a $44 \mathrm{kDa}$ protein in $E$. coli with spectral characteristics similar to $\mathrm{Vgb}$ and other globins. This haemoprotein (Hmp), product of the $b m p$ 
gene, has an $\mathrm{N}$-terminal haem domain that is $46 \%$ identical to $\mathrm{Vgb}$, with conservation of the putative haem ligands and the globin fold characteristic of $\mathrm{Vgb}$ and other globins. However, unlike $\mathrm{Vgb}, \mathrm{Hmp}$ has a Cterminal domain that is homologous to ferredoxin$\mathrm{NADP}^{+}$reductase and other members of a large family of proteins with highly conserved binding sites for $\mathrm{FAD}$ and $\mathrm{NAD}(\mathrm{P}) \mathrm{H}$ (Andrews et al., 1992; Karplus \& Bruns, 1994). Hmp may, therefore, be described as a flavohaemoglobin. Consistent with sequence analyses, purified Hmp contains haem B and FAD (Ioannidis et al., 1992; Cooper et al., 1994) and can be reduced with NADH in aerobic solution to yield a moderately stable oxygenated form (Ioannidis et al., 1992). The protein reacts with other ligands, including CO and cyanide; ESR spectroscopy of the nitrosyl complex indicates (Ioannidis $e t$ al., 1992) that the haem is attached to the protein through a nitrogenous ligand, probably the imidazole group of histidine-85, as suggested from sequence analyses (Vasudevan et al., 1991).

Despite its globin-like haem domain, Hmp shows the remarkable property of functioning as a soluble oxidase. The decay of the oxygenated form observed by Ioannidis et al. (1992) is due not to reversible dissociation of the haem-bound oxygen, but to oxygen reduction (Orii et al., 1992; Poole et al., 1994). In stopped-flow, rapid-scan experiments, the oxidized protein reacts rapidly with $\mathrm{NADH}$ in the presence of $130 \mu \mathrm{M}$ oxygen to form an oxygenated species, while the flavin remains largely oxidized. With excess NADH and limited oxygen, disappearance of the oxygenated species is accompanied by generation of the deoxy $\mathrm{Fe}$ (II) haem and flavin reduction. With limited NADH and excess oxygen, the NADH is consumed and both redox centres are oxidized. Superoxide anion is a major product of oxygen reduction (Orii et al., 1992; N. Ioannidis \& R. K. Poole, unpublished; R. K. Poole, R. D'mello \& Y. Orii, unpublished).

We have proposed, on the basis of these experiments (Poole et al., 1994), that Hmp could act as an oxygen sensor in E. coli by combining with intracellular oxygen, thus limiting flavin reduction in the aerobic steady state. Lowering of the cytoplasmic oxygen concentration would result in loss of the oxygenated species and extensive reduction of flavin. In view of the ability of Hmp to reduce $\mathrm{Fe}(\mathrm{III})$ (Andrews et al., 1992; Eschenbrenner et al., 1994; N. Ioannidis \& R. K. Poole, unpublished), such a mechanism might affect the redox status and thus activity of transcriptional regulators such as Fnr (Khoroshilova et al., 1995) or SoxR (Hidalgo \& Demple, 1994), both of which contain [Fe-S] clusters. This mechanism might also provide oxygen-modulated reduction of other substrates. However, we have recently shown (Vasudevan et al., 1995) that Hmp is predominantly cytoplasmic, and so any hypothesis that invokes oxygen reaction with Hmp must take into account the ability of Hmp to 'compete' for oxygen with the two terminal quinol oxidases in E. coli, cytochromes $b b^{\prime}$ (for an explanation of nomenclature, see Poole \& Chance, 1995) and $b d$ (Poole, 1994).

Consequently, in this paper, we characterize further the reactions of purified Hmp with NADH at very low oxygen tensions. We have also determined the $K_{\mathrm{m}}$ for oxygen during the oxidase reaction catalysed by Hmp to allow comparison with the $K_{\mathrm{m}}$ values for cytochromes $b o^{\prime}$ and $b d$, recently determined in one of our laboratories (D'mello et al., 1995, 1996).

\section{METHODS}

Organism, growth conditions and purification of Hmp. These procedures have been described in detail before (Vasudevan et al., 1991; Ioannidis $e t$ al., 1992). E. coli strain RSC521 harbours pPL341, containing the entire $b m p$ gene under the control of its own promoter cloned into pBR322. The strain was grown aerobically in Luria-Bertani medium (initial $\mathrm{pH} 7 \cdot 0$ ) supplemented with $0.2 \%(\mathrm{w} / \mathrm{v})$ glucose and ampicillin $\left(35 \mu \mathrm{g} \mathrm{m} \mathrm{m}^{-1}\right)$. Growth conditions in the 121 Biostat fermenter were described by Ioannidis et al. (1992). Cells were harvested when the $\mathrm{OD}_{600}$ of the culture (measured in a Pye-Unicam SP6-550 spectrophotometer, and after appropriate dilution to keep measured values below 0.6 in a $1 \mathrm{~cm}$ cuvette) was about 3 . Harvested cells, which were deep brown due to over-expression of the flavohaemoglobin, were washed and disrupted in a French pressure cell. Differential centrifugation removed cell debris and membranes, leaving an orange-red 'soluble' fraction. Hmp was purified by anion-exchange chromatography on DEAE-Sepharose CL-6B and gel filtration on Sephacryl S-200 exactly as described by Ioannidis et al. (1992). Protein concentrated by ultra-filtration was stored at, or below, $-70^{\circ} \mathrm{C}$ until used.

Stopped-flow spectrophotometry. The stopped-flow rapidscan spectrophotometer used (Orii, 1993; Poole et al., 1994) employs a diode array to record 512 absorption spectra over a $208 \mathrm{~nm}$ span, each in as little as $1.04 \mathrm{~ms}$. Timing of data collection is with reference to the instant when flow of the mixture stops.

Measurement of oxygen affinity. Oxygenated sperm whale oxymyoglobin (Sigma, no longer available) was a generous gift from Dr S. Hill (Nitrogen Fixation Laboratory, University of Sussex, UK). The procedure for preparation followed closely that of Appleby \& Bergersen (1980) and has been described before by D'mello et al. (1994, 1995). The concentration of myoglobin was determined by $\mathrm{CO}$ difference spectroscopy using the absorption coefficients cited by Wood (1984). Samples were diluted in potassium phosphate buffer $(50 \mathrm{mM}, \mathrm{pH} 7 \cdot 0)$ containing $1 \mathrm{mM}$ EDTA, which had been previously degassed with a $1 \%(\mathrm{v} / \mathrm{v})$ oxygen plus argon gas mixture (BOC). The procedure used the methods and calculations described by Appleby \& Bergersen (1980), except that the spectrophotometric measurements were modified as follows. A custombuilt (Radley's) glass optical cuvette $(1.3 \mathrm{ml})$ was completely filled with oxymyoglobin $(14 \mu \mathrm{M})$. Ascorbate $(1.1 \mathrm{mM})$ was included to scavenge superoxide, and $o$-dianisidine $(0 \cdot 12 \mathrm{mM})$ and horseradish peroxidase ( 6 Sigma units) were added to remove $\mathrm{H}_{2} \mathrm{O}_{2}$. The cuvette was sealed with a plastic plug having a fine hole through which Hmp and NADH (final concentrations $1.1 \mu \mathrm{M}$ and $0.32 \mathrm{mM}$, respectively) could be introduced. Deoxygenation of the myoglobin was continuously monitored by following the change in absorbance between 575 and $560 \mathrm{~nm}$, using a turbine-driven multi-wavelength time-sharing spectrophotometer (Chance et al., 1975). The absorbance difference (575 minus $560 \mathrm{~nm}$ ) was plotted against time and used to calculate oxygen consumption rates as free dissolved oxygen concentration declined. 


\section{RESULTS}

\section{Rapid-scan studies of the Hmp reaction with NADH and oxygen in oxygen-unsupplemented conditions}

The ability of Hmp to form an oxygenated compound at low oxygen concentrations was determined in buffers that had been sparged with oxygen-free nitrogen for $>15 \mathrm{~min}$ in the reservoir syringes of the stopped-flow apparatus. The oxygen concentration in the apparatus could not be assayed reliably, but an estimation of the residual oxygen concentration under these de-aerated conditions was made as follows. A solution of $\mathrm{Hmp}(2 \mu \mathrm{M}$ final concentration, i.e. after mixing), reduced with NADH (final concentration $0.5 \mathrm{mM}$ ), was sparged with nitrogen gas for $10 \mathrm{~min}$. Catalase was added to remove $\mathrm{H}_{2} \mathrm{O}_{2}$ and the sample was bubbled with nitrogen for a further $5 \mathrm{~min}$ to remove oxygen that might have resulted from the activity of catalase. The protein was mixed in the stopped-flow apparatus with an equal volume of nitrogen-bubbled buffer. The experiment was repeated using air-saturated buffer instead of nitrogen-bubbled buffer. In each case, difference spectra were plotted with the deoxy state attained at the end of the reaction as reference. The extent of formation of the oxygenated form, expressed as $\Delta A_{581-563}$, was 0.0186 in aerated buffer and 0.0024 for the de-aerated buffer. By assuming that the $K_{\mathrm{m}}$ for oxygen $(2 \cdot 6 \mu \mathrm{M}$; see later) can be equated with the dissociation constant for oxy Hmp, the free-oxygen concentration under the de-aerated conditions is $2.6 \times 0.0024 /(0 \cdot 0186-$ $0.0024)$ or $0.39 \mu \mathrm{M}$. This is consistent with previous estimates (Orii, 1993) of 'well below micromolar' based on the behaviour of myoglobin in a similar apparatus. Hereafter, the oxygen concentrations in the present experiments will be referred to as 'near-micromolar'.

When purified, Hmp is oxidized and the absolute absorption spectrum has a peak at $403.5 \mathrm{~nm}$ (Ioannidis $\mathrm{et} \mathrm{al}$.,

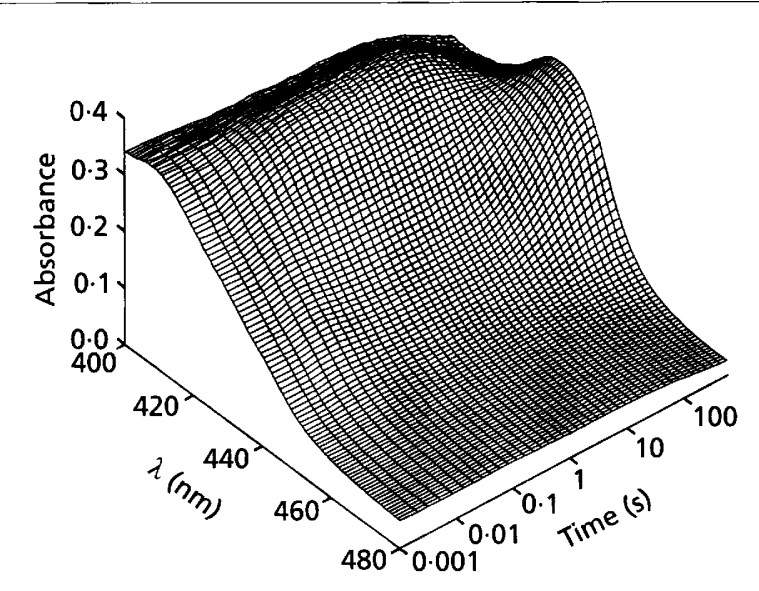

Fig. 1. Reaction of $\mathrm{Hmp}$ with $\mathrm{NADH}$ at near-micromolar oxygen concentration. De-aerated solutions of $\mathrm{Hmp}$ and $\mathrm{NADH}$ in $50 \mathrm{mM} \mathrm{NaCl}$ and $50 \mathrm{mM}$ Tris/ $\mathrm{HCl}$ buffer $(\mathrm{pH} \mathrm{8.0)}$ were mixed in the stopped-flow apparatus at $25^{\circ} \mathrm{C}$, giving final concentrations of $3.3 \mu \mathrm{M}$ haem, $0.5 \mathrm{mM}$ NADH and oxygen at near-micromolar concentration (Orii, 1993). Data collection for each spectrum $(208 \mathrm{~nm})$ took $1.04 \mathrm{~ms}$.
1992). It is stable in this form, becoming reduced only on addition of $\mathrm{NAD}(\mathrm{P}) \mathrm{H}$ or dithionite, for example. In the experiments reported here, as in our previous work, NADH was used as reductant because the affinity of Hmp for NADH $\left(K_{\mathrm{m}}\right.$ about $\left.2 \mu \mathrm{M}\right)$ is tenfold higher than that for NADPH (M. Anjum, N. Ioannidis \& R. K. Poole, unpublished). A nitrogen-gassed solution of the oxidized form was mixed in the stopped-flow apparatus with a nitrogen-sparged NADH solution in the second syringe to give final concentrations of $3.3 \mu \mathrm{M}$ haem and $0.5 \mathrm{mM}$ $\mathrm{NADH}$, as in our earlier work (Poole et al., 1994). The earliest spectrum recorded (1 ms after the mix) exhibited a peak at $407.8 \mathrm{~nm}$ which shifted to $409.9 \mathrm{~nm}$ at $10 \mathrm{~ms}$ and $412.5 \mathrm{~nm}$ after $100 \mathrm{~ms}$, with slight gains in intensity (see $0.001-0.1 \mathrm{~s}$ in three-dimensional plot; Fig. 1). The changes occurring after $10 \mathrm{~s}$ will be described below. $\mathrm{NADH}$ contributes to the high absorbance at the shortest wavelengths in Fig. 1, as revealed by scanning below $400 \mathrm{~nm}$ (not shown). These changes are similat to those observed with $130 \mu \mathrm{M}$ oxygen (Poole et al., 1994) and are attributed to formation of the oxygenated species, despite efforts to remove dissolved oxygen. The same procedure applied under similar experimental conditions was successful in keeping myoglobin in the deoxy state (Orii, 1993). Hmp appears, therefore, to have a high avidity for oxygen.

Difference spectra computed from the rapid-scan data allowed spectral changes occurring during selected time intervals to be visualized. With the $1 \mathrm{~ms}$ spectrum as reference (Fig. 2a), the changes in the first $13 \mathrm{~ms}$ are characterized by formation of a broad peak centred at $421 \mathrm{~nm}$, but with significant absorbance extending towards longer wavelengths, and an isosbestic point at $407 \mathrm{~nm}$. This result suggests a simultaneous increase in both oxygenated and deoxy forms, since the $421 \mathrm{~nm}$ peak is intermediate in position between the absorption maxima of the oxygenated $(413 \mathrm{~nm})$ and deoxy $(431.5 \mathrm{~nm})$ species (Ioannidis et al., 1992). A similar result was observed in the presence of $130 \mu \mathrm{M}$ oxygen (cf. Poole et al., 1994, Fig. 2a). However, the subsequent reaction progress at very low oxygen concentrations was markedly different. When the $12 \mathrm{~ms}$ spectrum was used as reference (Fig. 2b), the changes occurring between 12 and 1004 ms showed not only absorbance increases at $418 \mathrm{~nm}$, due to continued formation of the oxygenated form, but a distinct $440 \mathrm{~nm}$ trough with an isosbestic point at $430 \mathrm{~nm}$. The similarity of this trough position to that observed in difference spectra with the reduced state as a reference (e.g. CO plus reduced minus reduced, trough at $437 \mathrm{~nm}$; Ioannidis $e t$ al., 1992) strongly suggests that the oxygenated species is formed from the reduced form during the $12 \mathrm{~ms}$ to $1 \mathrm{~s}$ interval. Thus, the absorbance change at $440 \mathrm{~nm}$ during this period reflects conversion to the oxygenated form of the ferrous haem formed during the flow.

As seen in Fig. 1, the oxygenated form is stable for $<10 \mathrm{~s}$ under these oxygen-limited conditions; thereafter it decays to a species with a longer absorption maximum than can be attributed to the deoxy form. Difference spectra, in which the events occurring later than $10 \mathrm{~s}$ are plotted with the $10 \mathrm{~s}$ spectrum as reference (Fig. 2c), show 


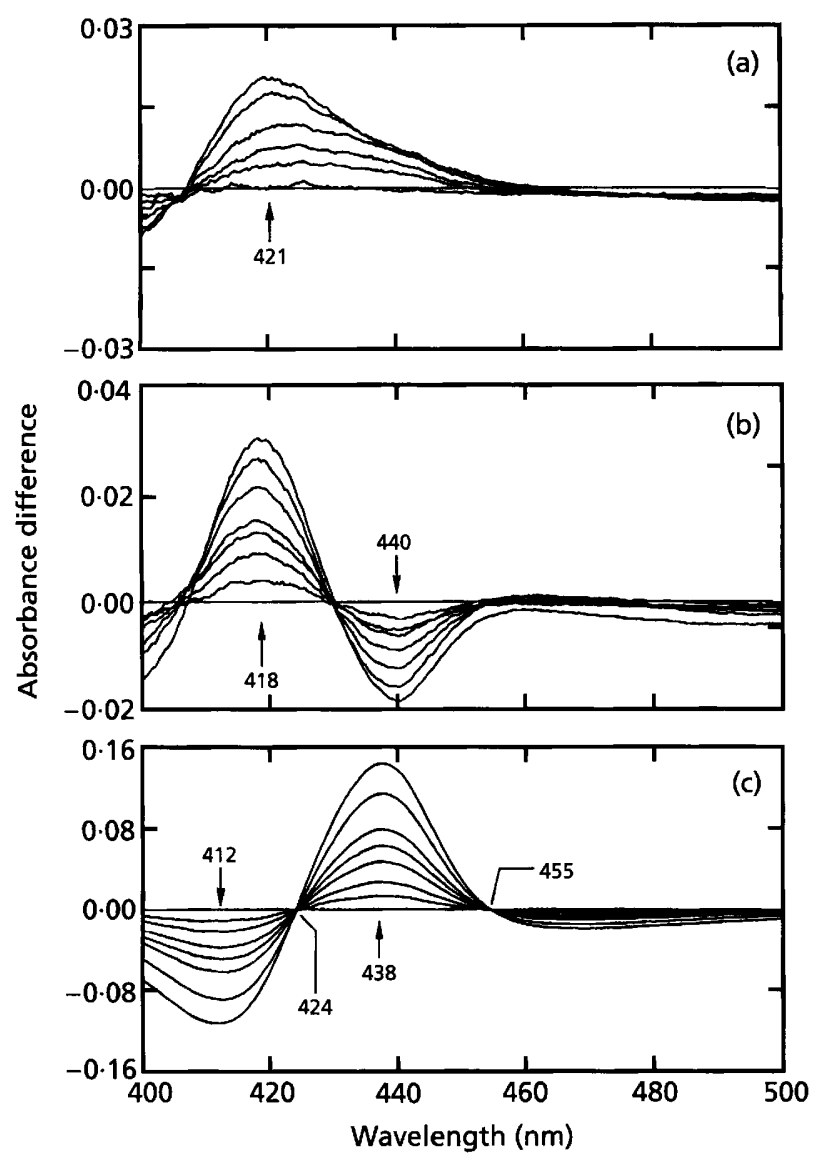

Fig. 2. Difference spectra obtained during the reaction of $\mathrm{Hmp}$ with NADH at near-micromolar oxygen concentration. Experimental conditions were as in Fig. 1. (a) Difference spectra at $1,3,4,5,7,11$ and $13 \mathrm{~ms}$, in the order indicated by the arrow, with the $1 \mathrm{~ms}$ spectrum as reference. (b) Difference spectra at 12, 16, 21, 25, 31, 51, 102 and $1004 \mathrm{~ms}$, in the order indicated by the arrows, with the $12 \mathrm{~ms}$ spectrum as reference. (c) Difference spectra at 10, 20, 30.5, 50.5, 71.2, 100.4, 203 and $500.7 \mathrm{~s}$, in the order indicated by the arrows, with the $10 \mathrm{~s}$ spectrum as reference.

an absorbance increase at $438 \mathrm{~nm}$ (the deoxy form) and a trough at $412 \mathrm{~nm}$ (loss of the oxygenated form). Isosbestic points were observed at 424 and $455 \mathrm{~nm}$.

Fig. 3 shows the absorbance changes recorded in Figs 1 and 2 as a function of time with either logarithmic (a) or linear (b) time scales. Comparison of these kinetic profiles with those obtained in the presence of a high concentration of oxygen (cf. Ioannidis et al., 1992, Fig. 1b) reveals the following. (1) A steady-state condition is attained within $200 \mathrm{~ms}$ of mixing in both cases, and the extent of formation of the oxygenated species $(417.7 \mathrm{~nm})$ is also similar in each case. (2) During the steady state, the level of the deoxy form $(439.7 \mathrm{~nm})$ is lower at nearmicromolar oxygen than in $130 \mu \mathrm{M}$ oxygen. (3) Significantly, under both conditions, the redox state of the flavin $(466.9 \mathrm{~nm})$ does not change in the interval between the first recording and the loss of the oxygenated form ( $\sim 10 \mathrm{~s}$ in Figs 1 and 3$)$, whereupon the signal decreases in amplitude, indicative of reduction.

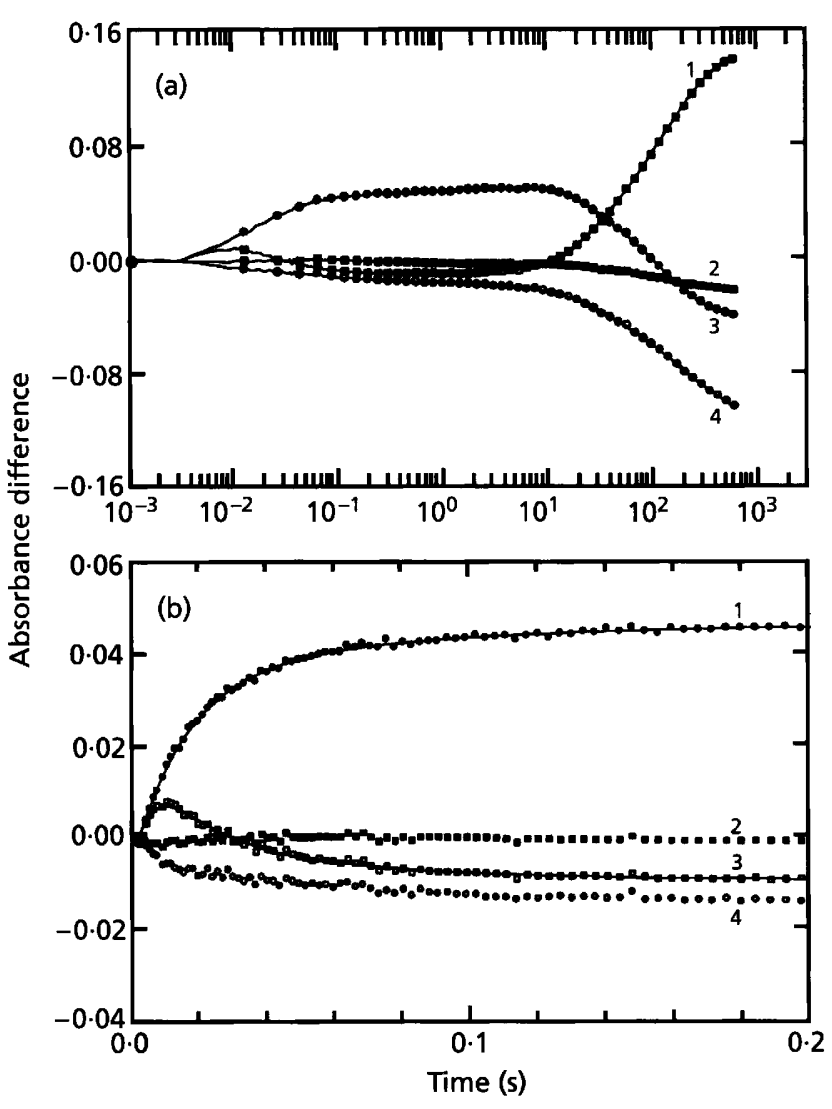

Fig. 3. Time courses for the reaction of $\mathrm{Hmp}$ with $\mathrm{NADH}$ at near-micromolar oxygen concentration. Experimental conditions were as in Fig. 1. (a) $1,431.1 \mathrm{~nm} ; 2,469.8 \mathrm{~nm} ; 3$, $412 \mathrm{~nm} ; 4,403.4 \mathrm{~nm}$. (b) $1,417.7 \mathrm{~nm} ; 2,466.9 \mathrm{~nm} ; 3,439.7 \mathrm{~nm}$; $4,402.6 \mathrm{~nm}$. Solid lines fitted to the symbols were obtained according to either a two-exponential $(417.7 \mathrm{~nm})$ or a threeexponential $(439.7 \mathrm{~nm})$ equation with the kinetic parameters given in Table 1.

Table 1. Apparent kinetic parameters for reduction of the flavohaemoglobin with NADH in the presence of a near-micromolar concentration of oxygen

Reciprocal relaxation times $\left(\mathrm{s}^{-1}\right)$ for the phases shown are given at four wavelengths. Standard errors for each kinetic parameter derived from the curve fitting are shown in parentheses. For details, see text.

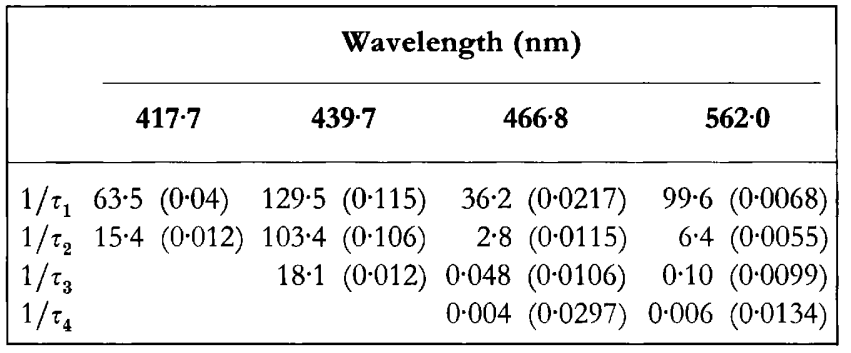

The absorbance increases for the oxygenated form formation are best explained by two exponential terms under both conditions. Table 1 lists the reciprocal relaxation 

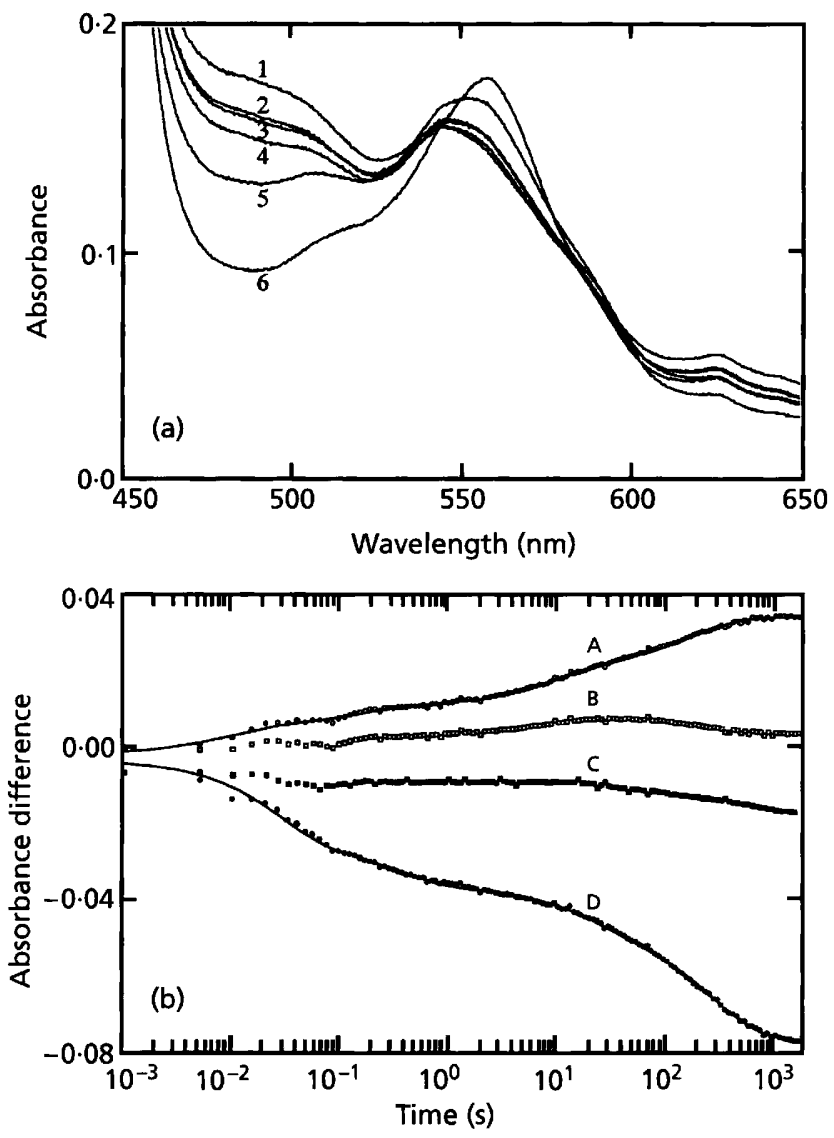

Fig. 4. Reaction of $\mathrm{Hmp}$ with $\mathrm{NADH}$ at near-micromolar oxygen concentration. De-aerated solutions of Hmp and NADH were mixed in the stopped-flow apparatus as described in the legend to Fig. 1, except that the final concentration of $\mathrm{Hmp}$ was $13 \mu \mathrm{M}$ haem. (a) Absolute spectra recorded at: 1, $1 \mathrm{~ms} ; 2,8 \mathrm{~ms} ; 3,21 \mathrm{~ms}$; $4,51 \mathrm{~ms} ; 5,20 \mathrm{~s} ; 6,1807 \mathrm{~s}$. (b) Absorbance changes at: $A, 562.0 \mathrm{~nm}$; B, $582.8 \mathrm{~nm} ;$ C, $625.9 \mathrm{~nm}$; D, $466.8 \mathrm{~nm}$. Solid lines fitted to the symbols were obtained according to a four-exponential equation with the kinetic parameters given in Table 1.

times for the $417 \cdot 7 \mathrm{~nm}$ change at near-micromolar oxygen concentrations; these values, $63.5 \mathrm{~s}^{-1}$ for the fast phase and $15.4 \mathrm{~s}^{-1}$ for the slower phase, should be compared with the corresponding parameters for $130 \mu \mathrm{M}$ oxygen, namely $111.2 \mathrm{~s}^{-1}$ and $17.7 \mathrm{~s}^{-1}$, respectively, calculated from Poole et al. (1994). Thus, only the initial phase is sensitive to oxygen concentration. The most significant quantitative difference is found between the traces for the deoxy form $(437.6 \mathrm{~nm}$ at high oxygen concentrations and $439.7 \mathrm{~nm}$ at low oxygen concentrations). The former is represented by a single exponential term $\left(1 / \tau_{1}=168 \mathrm{~s}^{-1}\right.$; Poole et al., 1994), whereas the latter is represented by three terms, i.e. $1 / \tau_{1}=129.5 \mathrm{~s}^{-1}, 1 / \tau_{2}=103.4 \mathrm{~s}^{-1}$ and $1 / \tau_{3}=18 \cdot 1 \mathrm{~s}^{-1}$ (Table 1 ). This result suggests that, at $130 \mu \mathrm{M}$ oxygen, the level of the oxygenated form stays unchanged after it is attained, but at low oxygen concentrations the deoxy form is generated transiently, giving way to the oxygenated form by reaction with oxygen (see Fig. 2b).

More stringent oxygen limitation was achieved by using a

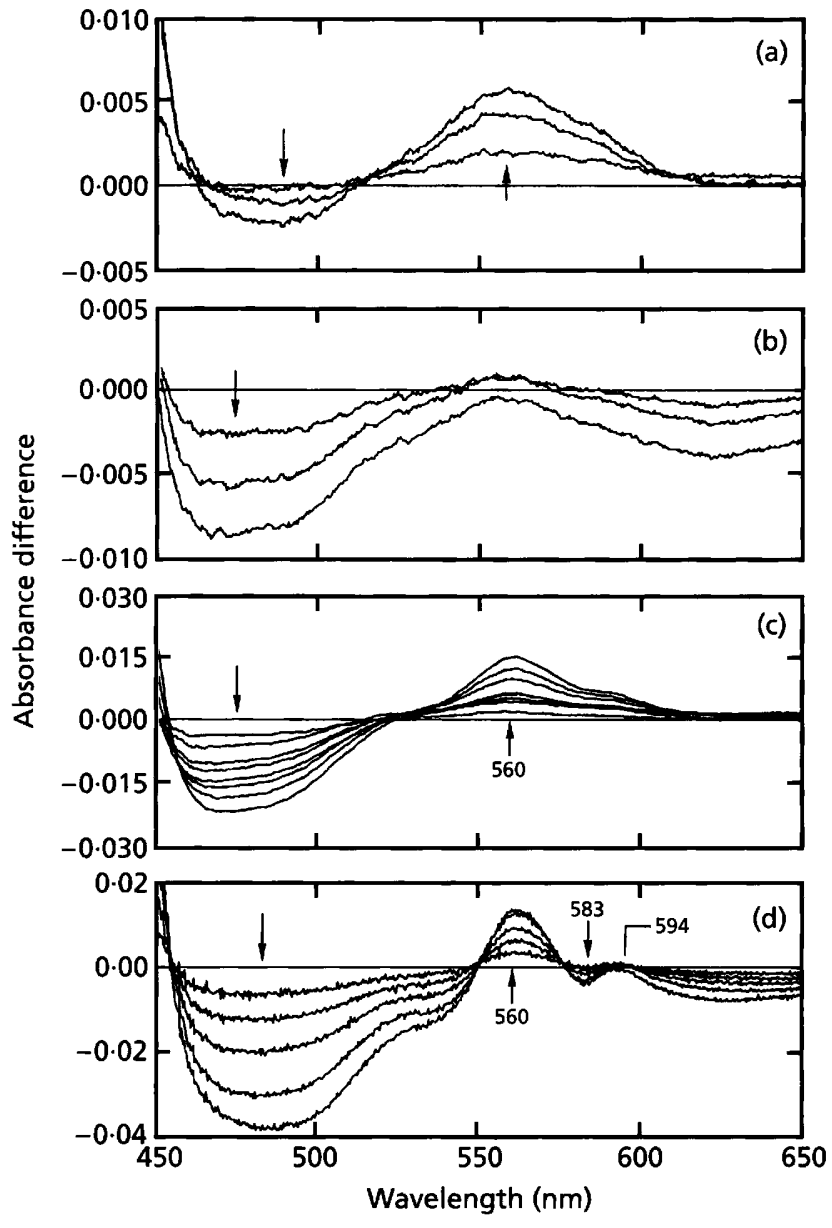

Fig. 5. Difference spectra obtained during the reaction of $\mathrm{Hmp}$ with $\mathrm{NADH}$ at near-micromolar oxygen concentration. Experimental conditions were as in Fig. 4. (a) Difference spectra at $8,11,16$ and $21 \mathrm{~ms}$, with the $8 \mathrm{~ms}$ spectrum as reference. (b) Difference spectra at 21, 30, 42 and $51 \mathrm{~ms}$, with the $21 \mathrm{~ms}$ spectrum as reference. (c) Difference spectra at $0.05,0.1,0.2$, $0.51,1.01,2.02,5.05,10.2$ and $20 \mathrm{~s}$, with the $0.05 \mathrm{~s}$ spectrum as reference. (d) Difference spectra at 20,50.4, 101, 203, 508 and $1807 \mathrm{~s}$, with the $20 \mathrm{~s}$ spectrum as reference. Labelled arrows indicate wavelengths of features referred to in the text. Unlabelled arrows show the direction of change of broad spectral regions.

higher protein concentration $(13 \mu \mathrm{M}$ haem), which also permitted observation in the $\alpha / \beta$ regions of the spectrum. Formation of the oxygenated species was still evident in the $1 \mathrm{~ms}$ spectrum (Fig. 4a). Up to $51 \mathrm{~ms}$ after the start of the reaction, the absolute spectra revealed little change in the level of the oxygenated form and little flavin reduction. By about $20 \mathrm{~ms}$ (scan 3 in Fig. $4 \mathrm{a}$ ), the $\beta$-band had shifted to $558 \mathrm{~nm}$, characteristic of the deoxy form (Ioannidis et al., 1992), and flavin reduction was evident from the trough below $500 \mathrm{~nm}$. Fig. 4(b) presents these absorbance changes on a logarithmic time scale. The subsequent spectral changes were complex, suggesting multiple reaction steps, and were further analysed by plotting time difference spectra (Fig. 5). Between 8 and 21 ms (Fig. 5a), the time difference spectra revealed an absorbance increase centred at $560 \mathrm{~nm}$ and a small absorbance decrease centred 


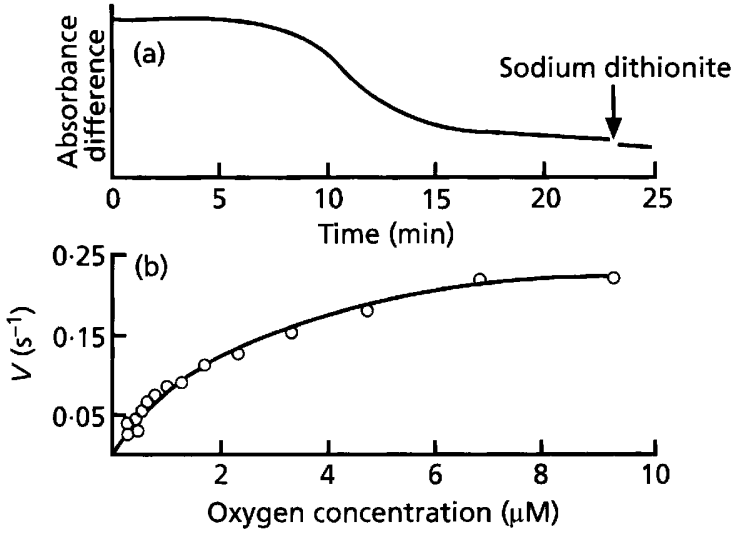

Fig. 6. Oxygen affinity of the $\mathrm{NADH}$ oxidase activity of $\mathrm{Hmp}$ measured by deoxygenation of oxymyoglobin. (a) Progress of deoxygenation measured at 560 minus $575 \mathrm{~nm}$ in the dualwavelength spectrophotometer. Additions are shown. (b) Oxygen dependence of oxidase activity. Experimental conditions are described in the text.

at about $490 \mathrm{~nm}$. The breadth of the band suggests formation of both oxygenated and deoxygenated forms, as revealed in the Soret region (Fig. 2). The peak height was larger than the trough depth, attributable to the reduction of haem $B$ without accompanying FAD reduction. Between 21 and $51 \mathrm{~ms}$ (Fig. 5b), the absorbance decrease centred around $470 \mathrm{~nm}$ was the main change, indicating the beginning of reduction of FAD. From $50 \mathrm{~ms}$ to $20 \mathrm{~s}$ (Fig. 5c), the absorbance increased with a maximal change at $560 \mathrm{~nm}$ but extending to $600 \mathrm{~nm}$. The absorbance decrease at $470 \mathrm{~nm}$ (flavin reduction) continued. Finally, in the 20-1807 s interval (Fig. 5d), a small portion of the oxygenated form (decreasing absorbance at $583 \mathrm{~nm}$ and about $540 \mathrm{~nm}$ ) was converted into the deoxy form $(560 \mathrm{~nm})$, also accompanied by further FAD reduction. In accordance with these complex changes, the absorbance changes at both $562.0 \mathrm{~nm}$ for haem B and $466.8 \mathrm{~nm}$ for FAD are each simulated by four exponential terms. The reciprocals of relaxation times (Table 1) for the $466.8 \mathrm{~nm}$ trace were lower than those for the $562.0 \mathrm{~nm}$ trace at each phase, suggesting that haem $B$ is reduced prior to FAD. This may reflect rapid intramolecular electron transfer from FAD to haem, as invoked in an earlier model to explain the predominantly oxidized state of FAD during the oxygenated steady state.

\section{Oxygen affinity of Hmp during NADH oxidation}

Fig. 6(a) shows the kinetics of deoxygenation of oxymyoglobin resulting from the consumption of 'free' dissolved oxygen by Hmp with NADH as reductant. Spectra recorded before and after the deoxygenation (not shown) revealed that conversion of oxymyoglobin to the ferric form, rather than the deoxygenated form, made a negligible contribution to the absorbance changes measured, as we have observed previously (D'mello et al., 1994, 1995). The addition of dithionite on completion of Hmpcatalysed deoxygenation resulted in a negligible further change in absorbance (Fig. 6a). The rates of oxygen consumption in a typical experiment are plotted against oxygen concentration in Fig. 6(b). The $K_{\mathrm{m}}$ was $2.6 \pm 0.16 \mu \mathrm{M}$ and the $V_{\max }$, expressed as a turnover number, was $18 \pm 0 \cdot 48 \mathrm{~min}^{-1}$ (each a mean of four separate determinations).

\section{DISCUSSION}

Proteins having haem domains that show clear homologies to the well-characterized oxygen-carrying globins of multicellular organisms have been described in several micro-organisms. Small proteins $(12.5-15 \mathrm{kDa})$ that resemble superficially the well-characterized Vitreoscilla globin have been reported in protozoa (Takagi, 1993) and the cyanobacterium Nostoc (Potts et al., 1992). Larger, two-domain or chimaeric proteins that resemble Hmp have been reported in yeasts (Iwaasa $\mathrm{et}$ al., 1992; Zhu \& Riggs, 1992) and several bacteria, including Alcaligenes eutrophus (Probst et al., 1979; Cramm et al., 1994) and Erwinia chrysanthemi (Favey et al., 1995). The function(s) of none of these proteins is known. The reactions of microbial globins with oxygen have received scant attention, even though the ligand-binding characteristics should yield important clues to function(s). Most, but not all (e.g. Favey et al., 1995), of the above microbial globins have been shown to bind oxygen. Apart from work on Vgb (Webster, 1987), only the yeast globin has been extensively studied in this respect. Oshino et al. (1973a) showed that the oxygenated form of yeast haemoglobin could be detected in intact cells of Candida mycoderma and that the oxygen concentration producing half-oxygenation of isolated globin in vivo was $0.015-0.02 \mu \mathrm{M}$. The dissociation rate constant was $17 \mathrm{~s}^{-1}\left(\mathrm{pH} 7 \cdot 0,23^{\circ} \mathrm{C}\right)$, very similar to the values of animal globins and leghaemoglobin (Oshino et al., 1973b).

A striking feature of $\mathrm{Hmp}$ is its ability to act as an oxidase, i.e. Hmp not only binds oxygen, but reduces it to superoxide (Orii et al., 1992; M. Anjum, N. Ioannidis \& R. K. Poole, unpublished; R. K. Poole, R. D'mello \& Y. Orii, unpublished). Vgb is also an oxidase, being first described as 'a soluble cytochrome $o^{\prime}$ (' 0 ' for oxidase; Webster \& Liu, 1974). Thus, an important aspect of understanding the physiological function of Hmp (and presumably its homologues in other organisms) must be to determine the ability of Hmp to 'compete' for oxygen with other oxygen-consuming enzymes. In E. coli (and probably the other enteric bacteria in which Hmp-like proteins have been tentatively found; see Andrews $e t$ al., 1992), there are two such oxidases, cytochrome $b o^{\prime}$ and cytochrome $b d$. The former is a member of the haemcopper super-family of membrane-bound terminal oxidases that includes mitochondrial cytochrome $c$ oxidase. Cytochrome $b o^{\prime}$ is a proton pump, whose synthesis is markedly affected by growth conditions (Cotter et al., 1990 ) such that it is present maximally in cells grown with 'high aeration'. Recent determinations (D'mello et al., 1995) of the affinity of cytochrome $b o^{\prime}$ for oxygen, using the same sensitive technique as described in the present work, have revealed $K_{\mathrm{m}}$ values between 0.016 and 
$0.35 \mu \mathrm{M}$. The alternative oxidase, cytochrome $b d$, is structurally unrelated to cytochrome $b o^{\prime}$, in having a haem-haem binuclear centre where oxygen is reduced, and is synthesized under microaerobic conditions. Several measurements of its affinity for oxygen (e.g. Rice \& Hempfling, 1978) have suggested that its affinity for oxygen is much higher than that of cytochrome $b o^{\prime}$, suiting it to function as a scavenger of oxygen. Recently, we have used the deoxygenation of oxyleghaemoglobin to demonstrate a remarkably high affinity for oxygen of around $5 \mathrm{nM}$ (D'mello et al., 1996), unprecedented for a terminal oxidase. It is tacitly assumed, though not formally proven, that the oxygen-consuming sites of both oxidases are on the inner, cytoplasmic face of the membrane, although some experimental evidence with Azotobacter vinelandii is consistent with the view that cytochrome $b d$ might reduce oxygen on the outer side of the membrane (Jones, 1977; D'mello et al., 1994). Thus, even the lower affinity oxidase, cytochrome $b 0^{\prime}$, has a $K_{m}$ well below that of Hmp. If Hmp (Vasudevan et al., 1995) and the oxygen-consuming site of cytochrome $b o^{\prime}$ are in the cytoplasm, and assuming that access of oxygen to the cytoplasm is unrestricted (Unden et al., 1995) and that there are no significant intracellular gradients of oxygen concentration, the oxidase activity of $\mathrm{Hmp}$ is unlikely to make a major contribution to cellular oxygen uptake. Reinforcing this view are the relative intracellular concentrations of the oxidases and Hmp. Cotter et al. (1990) have calculated that the concentrations of cytochromes $b o^{\prime}$ and $b d$ are 2-300 and 200-600 molecules per cell, respectively, according to growth conditions, and are readily measured in intact cell suspensions. In contrast, Hmp is undetectable in intact cells or crude extracts, unless over-expressed from plasmid-borne bmp (Vasudevan et al., 1991) or induced by paraquat (M. Anjum, N. Ioannidis \& R. K. Poole, unpublished). We conclude, therefore, that under normal growth conditions Hmp does not contribute substantially to cellular uptake of oxygen in E. coli.

It is striking, however, that even the use of nitrogensparged reactants and relatively high protein concentrations do not prevent the formation of the oxygenated species of Hmp when the ferric form is mixed with $\mathrm{NADH}$ in the stopped-flow apparatus. Under the experimental conditions employed here, the oxygen concentration at the instant of initiating the reaction is estimated to be near-micromolar (Orii, 1993), declining thereafter as a consequence of the oxidase activity of Hmp. We are not aware of any estimates of the intracellular concentration of oxygen in $E$. coli, but it is reasonable to suppose that Hmp will be substantially oxygenated in vivo.

This conclusion is immediately relevant to our previous proposal (Poole et al., 1994) that Hmp may act as an oxygen sensor. In that model, occupancy of the haem by oxygen is envisaged to control the steady-state reduction level of FAD and the transfer of electrons from FAD to an (unknown) acceptor. In the presence of oxygen, the oxygenated complex is formed and the flavin is predominantly oxidized; in the absence of oxygen, e.g. as a result of oxygen consumption by Hmp, the flavin is predomin- antly reduced and allows Hmp to act as a reductase for, perhaps, $\mathrm{Fe}(\mathrm{III})$. The results presented in this paper demonstrate that a key element in this model, namely the control of flavin oxidoreduction by oxygen binding, is operative at or below micromolar concentrations of oxygen. The data do not, however, distinguish between models that invoke (a) rapid electron withdrawal from FAD by intramolecular electron transfer or (b) conformational changes in the protein elicited by occupancy of ligand at the haem and subsequent effects on flavin chemistry (Cooper et al., 1994). It is interesting to note that in the FixL protein of Rbizobium meliloti, GillesGonzález et al. (1995) have shown that the presence of any ligand that gives a high-spin state of the haem endows the protein with kinase activity, which initiates a cascade of reactions culminating in nitrogen fixation. Oxygen binds to FixL, giving a low-spin complex that prevents induction of nitrogen fixation. The effects of other ligands on the reductase activities of $\mathrm{Imp}$ remain to be established.

Detailed kinetic studies of oxygen binding and reduction by Hmp remain to be performed. The present data reveal that an early stage in the formation of the oxygenated species is sensitive to oxygen concentration and that persistence of the oxygenated form at low oxygen concentrations involves reduction of Hmp by NADH, followed by oxygenation on a time scale that can be observed using the rapid-scan technique. The kinetics of oxygen binding to, and dissociation from, Hmp are important objectives of future work.

\section{ACKNOWLEDGEMENTS}

R.K.P. thanks BBSRC (SERC) for Research Grants (GR/G 13761 and GR/H 22265). Y.O. thanks the Ministry of Education, Science and Culture, Japan, for a Grant-in-Aid for Scientific Research on Priority Areas of 'Cellular Energy' (no. 04266105). We are grateful to Dr Rita D'mello for assistance with the oxygen affinity measurements and Dr Susan Hill for stimulating discussions and a gift of oxymyoglobin. The continucd interest and support of Dr Cyril Appleby in these studies is gratefully acknowledged.

\section{REFERENCES}

Andrews, S. C., Shipley, D., Keen, J. N., Findlay, J. B. C., Harrison, P. M. \& Guest, J. R. (1992). The haemoglobin-like protein (HMP) of Escherichia coli has ferrisiderophore reductase activity and its $\mathrm{C}$ terminal shares homology with ferredoxin NADP + reductases. FEBS Lett 302, 247-252.

Appleby, C. A. \& Bergersen, F. J. (1980). Preparation and experimental use of leghaemoglobin. In Methods of Evaluating Biological Nitrogen Fixation, pp. 315-335. Edited by F. J. Bergersen. Chichester: John Wiley.

Boerman, S. \& Webster, D. A. (1982). Control of heme content in Vitreoscilla by oxygen. J Gen Appl Microbiol 28, 35-43.

Chance, B., Legallais, V., Sorge, J. \& Graham, N. (1975). A versatile time-sharing multichannel spectrophotometer, reflectometer, and fluorometer. Anal Biochem 66, 498-514.

Cooper, C. E., loannidis, N., D'mello, R. \& Poole, R. K. (1994). Haem, flavin and oxygen interactions in Hmp, a flavohaemoglobin from Lischerichia coli. Biochem Soc Trans 22, 709-713. 
Cotter, P. A., Chepuri, V., Gennis, R. B. \& Gunsalus, R. P. (1990). Cytochrome $o$ (cyo $A B C D E)$ and $d(c y d A B)$ oxidase gene expression in Escherichia coli is regulated by oxygen, $\mathrm{pH}$, and the fnr gene product. J Bacteriol 172, 6333-6338.

Cramm, R., Siddiqui, R. A. \& Friedrich, B. (1994). Primary sequence and evidence for a physiological function of the flavohaemoprotein of Alcaligenes eutrophus. J Biol Chem 269, 7349-7354.

D'mello, R., Hill, S. \& Poole, R. K. (1994). Determination of the oxygen affinities of terminal oxidases in Azotobacter vinelandii using the deoxygenation of oxyleghaemoglobin and oxymyoglobin: cytochrome $b d$ is a low-affinity oxidase. Microbiology $140,1395-1402$.

D'mello, R., Hill, S. \& Poole, R. K. (1995). The oxygen affinity of cytochrome $b o^{\prime}$ in Escherichia coli determined by the deoxygenation of oxyleghemoglobin and oxymyoglobin; $K_{\mathrm{m}}$ values for oxygen are in the submicromolar range. $J$ Bacteriol 177, 867-870.

D'mello, R., Hill, S. \& Poole, R. K. (1996). The cytochrome $b d$ quinol oxidase in Escherichia coli has an extremely high oxygen affinity and two oxygen-binding haems: implications for regulation of activity in vivo by oxygen inhibition. Microbiology 142, (in press).

Eschenbrenner, M., Coves, J. \& Fontecave, M. (1994). Ferric reductases in Escherichia coli: the contribution of the haemoglobinlike protein. Biochem Biophys Res Commun 198, 127-131.

Favey, S., Labesse, G., Vouille, V. \& Boccara, M. (1995). Flavohaemoglobin $\mathrm{HmpX}$ : a new pathogenicity determinant in Erwinia cbrysanthemi strain 3937. Microbiology 141, 863-871.

Gilles-González, M. A., González, G. \& Perutz, M. F. (1995). Kinase activity of oxygen sensor FixL depends on the spin state of its heme iron. Biochemistry 34, 232-236.

Hidalgo, E. \& Demple, B. (1994). An iron-sulfur center essential for transcriptional activation by the redox-sensing SoxR protein. EMBO J 13, 138-146.

loannidis, N., Cooper, C. E. \& Poole, R. K. (1992). Spectroscopic studies on an oxygen-binding haemoglobin-like flavohaemoprotein from Escherichia coli. Biocbem J 288, 649-655.

Iwaasa, H., Takagi, T. \& Shikama, K. (1992). Amino acid sequence of yeast hemoglobin. A two-domain structure. J Mol Biol 227, 948-954.

Jones, C. W. (1977). Aerobic respiratory systems in bacteria. In Microbial Energetics, pp. 23-59. Edited by B. A. Haddock \& W. A. Hamilton. Cambridge: Cambridge University Press.

Karplus, P. A. \& Bruns, C. M. (1994). Structure-function relation for ferredoxin reductase. J Bioenerg Biomembr 26, 89-99.

Khoroshilova, N., Beinert, H. \& Kiley, P. J. (1995). Association of a polynuclear iron-sulfur center with a mutant FNR protein enhances DNA binding. Proc Natl Acad Sci US A 92, 2499-2503.

Khosla, C. \& Bailey, J. E. (1988). Heterologous expression of a bacterial haemoglobin improves the growth properties of recombinant Escherichia coli. Nature 331, 633-635.

Khosravi, M., Webster, D. A. \& Stark, B. C. (1990). Presence of the bacterial hemoglobin gene improves $\alpha$-amylase production of a recombinant Escherichia coli strain. Plasmid 24, 190-194.

Orii, Y. (1993). Immediate reduction of cytochrome $c$ by photoexcited NADH: reaction mechanism as revealed by flow-flash and rapid-scan studies. Biochemistry 32, 11910-11914.

Orii, Y. \& Webster, D. A. (1986). Photodissociation of oxygenated cytochrome $o(\mathrm{~s})$ (Vitreoscilla) and kinetic studies of reassociation. J Biol Chem 261, 3544-3547.

Orii, Y., loannidis, N. \& Poole, R. K. (1992). The oxygenated flavohaemoglobin from Escherichia coli: evidence from photodiss- ociation and rapid-scan studies for two kinetic and spectral forms. Biochem Biophys Res Commun 187, 94-100.

Oshino, R., Oshino, N., Chance, B. \& Hagihara, B. (1973a). Studies on yeast hemoglobin. The properties of yeast hemoglobin and its physiological function in the cell. Eur J Biochem 35, 23-33.

Oshino, R., Asakura, T., Tajio, K., Oshino, N., Chance, B. \& Hagihara, B. (1973b). Purification and molecular properties of yeast hemoglobin. Eur J Biochem 39, 581-590.

Perutz, M. F. (1986). A bacterial haemoglobin. Nature 322, 405.

Poole, R. K. (1994). Oxygen reactions with bacterial oxidases and globins: binding, reduction and regulation. Antonie Leeuwenboek 65, 289-310.

Poole, R. K. \& Chance, B. (1995). Oxidase names: to ' 3 ' or not to '3'? Microbiology 141, 752-753.

Poole, R. K., Ioannidis, N. \& Orii, Y. (1994). Reactions of the Eschericbia coli flavohaemoglobin (Hmp) with oxygen and reduced nicotinamide adenine dinucleotide: evidence for oxygen switching of flavin oxidoreduction and a mechanism for oxygen sensing. Proc $R$ Soc Lond B 255, 251-258.

Potts, M., Angeloni, S. V., Ebel, R. E. \& Bassam, D. (1992). Myoglobin in a cyanobacterium. Science 256, 1690-1692.

Probst, I., Wolf, G. \& Schlegel, H. G. (1979). An oxygen-binding flavohemoprotein from Alcaligenes eutrophus. Biocbim Biopbys Acta 576, 471-478.

Rice, C. W. \& Hempfling, W. P. (1978). Oxygen-limited continuous culture and respiratory energy conservation in Escherichia coli. $J$ Bacteriol 134, 115-124.

Takagi, T. (1993). Hemoglobins from single-celled organisms. Curr Opin Struct Biol 3, 413-418.

Unden, G., Becker, S., Bongaerts, J., Holighaus, G., Schirawski, J. \& Six, S. (1995). $\mathrm{O}_{2}$-sensing and $\mathrm{O}_{2}$-dependent gene regulation in facultatively anaerobic bacteria. Arch Microbiol 164, 81-90.

Vasudevan, S. G., Armarego, W. L. F., Shaw, D. C., Lilley, P. E. \& Poole, R. K. (1991). Isolation and nucleotide sequence of the $b m p$ gene that encodes a haemoglobin-like protein in Escherichia coli K-12. Mol Gen Genet 226, 49-58.

Vasudevan, S. G., Tang, P., Dixon, N. E. \& Poole, R. K. (1995). Distribution of the flavohaemoglobin, HMP, between periplasm and cytoplasm in Eschericbia coli. FEMS Microbiol Lett 125, 219-224.

Wakabayashi, S., Matsubara, H. \& Webster, D. A. (1986). Primary sequence of a dimeric haemoglobin from Vitreoscilla. Nature 322, 481-483.

Webster, D. A. (1987). Structure and function of bacterial hemoglobin and related proteins. In Advances in Inorganic Chemistry, vol. 7, pp. 245-265. Edited by G. L. Eichhorn \& L. G. Marzilli. New York: Elsevier.

Webster, D. A. \& Liu, C. Y. (1974). Reduced nicotinamide adenine dinucleotide cytochrome $\theta$ reductase associated with cytochrome 0 purified from Vitreoscilla. J Biol Chem 249, 4257-4260.

Wood, P. M. (1984). Bacterial proteins with CO-binding $b$ - or $c$ type haem. Functions and absorption spectroscopy. Biochim Biophys Acta 768, 293-317.

Zhu, H. \& Riggs, A. F. (1992). Yeast flavohemoglobin is an ancient protein related to globins and a reductase family. Proc Natl Acad Sci US A 89, 5015-5019.

Received 3 November 1995; revised 5 February 1996; accepted 8 February 1996. 